\title{
Opioid-induced gut microbial disruption and bile dysregulation leads to gut barrier compromise and sustained systemic inflammation
}

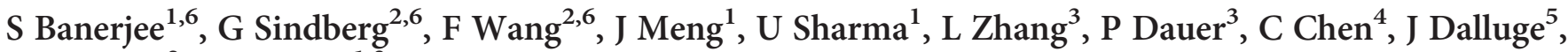 \\ T Johnson ${ }^{2}$ and S Roy ${ }^{1,3}$
}

Morphine and its pharmacological derivatives are the most prescribed analgesics for moderate to severe pain management. However, chronic use of morphine reduces pathogen clearance and induces bacterial translocation across the gut barrier. The enteric microbiome has been shown to have a critical role in the preservation of the mucosal barrier function and metabolic homeostasis. Here, we show for the first time, using bacterial 16s rDNA sequencing, that chronic morphine treatment significantly alters the gut microbial composition and induces preferential expansion of Gram-positive pathogenic and reduction in bile-deconjugating bacterial strains. A significant reduction in both primary and secondary bile acid levels was seen in the gut, but not in the liver with morphine treatment. Morphine-induced microbial dysbiosis and gut barrier disruption was rescued by transplanting placebo-treated microbiota into morphinetreated animals, indicating that microbiome modulation could be exploited as a therapeutic strategy for patients using morphine for pain management.

\section{INTRODUCTION}

Despite being the predominant drug of choice for moderate to chronic pain management, morphine treatment results in severe comorbidities because of peripheral side effects. In the past few years, several groups including ours have been actively working on understanding the phenomenon and delineating the mechanism underlying peripheral effects of morphine on immune cells and its role in exacerbating comorbidities associated with its use or abuse. The emerging consensus from all these studies conclusively demonstrate that opioid drugs cause adverse effects, including increased predisposition to infection, exacerbating pathogenesis, impairing endotoxin tolerance, and more recently inducing gut barrier disruption and bacterial translocation. ${ }^{1-5}$ The mammalian gut houses a robust and resilient microbiota that maintains a high degree of species diversity-the most important defense against pathogen virulence and invasion. ${ }^{6}$ In diseased states, the microbial balance that favors homeostasis is perturbed, resulting in a loss in the richness and diversity of the bacterial components. Any significant shift in the composition of the microbiota ("dysbiosis") favors the appearance of distinct pathogens and has been implicated in the pathogenesis of diverse illnesses, such as obesity, type 2 diabetes, inflammatory bowel disease, and cardiovascular disease. ${ }^{7-9}$ However, until now there have been no studies investigating how morphine treatment modulate the gut microbiota and its contribution to morphine-induced pathology such as microbial translocation and systemic immune activation. Furthermore, a cyclical relationship exists between gut microbial homeostasis and healthy hepatoenteric circulation of host metabolites, particularly bile acid. Change in gut microbial composition (microbial dysbiosis) leading to bile acid changes has been correlated with gut barrier disruption and inflammation. ${ }^{10-12}$ Systemic insults such as opioid use/abuse, which elevate systemic inflammation,

\footnotetext{
${ }^{1}$ Department of Surgery and Pharmacology, Division of Infection, Inflammation and Vascular Biology, University of Minnesota, Minneapolis, Minnesota, USA. ${ }^{2}$ Department of Veterinary Medicine, University of Minnesota, Minneapolis, Minnesota, USA. ${ }^{3}$ Department of Pharmacology, University of Minnesota, Minneapolis, Minnesota, USA. ${ }^{4}$ Department of Food Science and Nutrition, University of Minnesota, Minneapolis, Minnesota, USA and ${ }^{5}$ Department of Chemistry, University of Minnesota, Minneapolis, Minnesota, USA. Correspondence: S Roy (royxx002@umn.edu)

${ }^{6}$ The first three authors contributed equally to this work.
} 
would be expected to alter the gut microbial composition (microbial dysbiosis) and induce bile acid changes. The linearity and order of events needs to be determined for designing any kind of clinical intervention. Furthermore, proinflammatory environment and infiltration of immune cells in the gut tissues is strongly correlated to the maintenance of the pathogenic phenotype.

In this study, we establish a link between the two phenomena, namely gut barrier compromise and dysregulated bile acid metabolism. We show for the first time that morphine fosters significant gut microbial dysbiosis and disrupts cholesterol/bile acid metabolism. Changes in the gut microbial composition is strongly correlated to disruption in host inflammatory homeostasis, ${ }^{13,14}$ and in many diseases (e.g., cancer/HIV infection), persistent inflammation is known to aid and promote the progression of the primary morbidity. We show here that chronic morphine, gut microbial dysbiosis, disruption of cholesterol/bile acid metabolism, and gut inflammation have a linear correlation. This opens up the prospect of devising minimally invasive adjunct treatment strategies involving microbiome and bile acid modulation and thus bringing down morphine-mediated inflammation in the host.

\section{RESULTS}

\section{Morphine induces global changes in gut microbiota}

We have previously shown that bacterial translocation because of the gut mucosal barrier compromise are derived from the gut, mostly from commensal flora. ${ }^{3,15}$ In this report, morphine ( $\sim 1 \mu \mathrm{M}$ serum concentration) was administered into C57B16/j mice in analgesic doses and intestinal fecal contents were collected $72 \mathrm{~h}$ later and analyzed for microbial composition. While nonsignificant changes were observed in $\alpha$-diversity among treatment groups (Figure 1a), a significant shift in gut microbial composition between placebo- and morphinetreated animals was observed (principal coordinate analysis $(\mathrm{pCoA})$; Figure 1b). Microbiome of the animals treated with both morphine and naltrexone ( $\mu$-opioid receptor antagonist) clustered with the placebo-treated animals (Figure 1c). Analysis of the significant changes in $\beta$-diversity because of morphine shows a net expansion of Gram-positive Firmicutes compared with all other major phyla of gut bacteria (Figure 1d). Of the 5585 cumulative operational taxonomic units (OTUs) detected among placebo- and morphineimplanted animals, 117 OTUs (within 30 families) were found to be significantly different between the groups. Top OTUs from each family with higher (red) or lower (blue) relative abundance were used to compute an idealized tree using taxonomic classifications (Figure 1e). In this heat map, 28 out of 34 OTUs belong to phylum Firmicutes with a net preferential expansion in the morphine-treated animals. Reduction in comparative abundance was observed for phylum Bacteroidetes in the morphine-treated animals, thus reducing the Bacteroidetes/Firmicutes ratio in those animals (Supplementary Figure S1 online). Similar changes in the Bacteroidetes/Firmicutes ratio is correlated with increased systemic inflammation in obesity and aging. ${ }^{9,16,17}$ Additionally, elevated abundance of bacterial families Enterococcaceae, Staphylococcaceae, Bacillaceae, Streptococcaceae, and Erysipelotrichaceae was seen in the morphine-implanted animals, all belonging to the phylum Firmicutes. In this context, we and others have shown that chronic morphine increases the susceptibility ${ }^{18,19}$ and rate of pathogenesis ${ }^{18,20}$ of mucosal infections, leading to sepsis and septic shock ${ }^{5,21,22}$ with significant contribution of Gram-positive bacteria in morphine-induced polymicrobial sepsis. ${ }^{23}$

\section{Role of TLR2 and $\mu$-opioid receptor in morphine-induced microbial dysbiosis}

Literature, ${ }^{5,22}$ including our own published works, ${ }^{2-4,19}$ show that morphine-mediated gut microbial dysbiosis and mucosal barrier compromise results in bacterial translocation (predominantly Gram-positive), leading to localized gut and systemic inflammation. Interestingly, the translocated bacteria, serotyped from the liver of morphine-treated animals, ${ }^{3}$ belonged to the classes Staphylococcaceae, Enterococcaceae, and Bacillaceae, all part of the commensal flora and the same classes that exhibit preferential expansion in the gut microbiome upon morphine treatment (Figure 1d and Supplementary Figure S2). Hence, we wanted to verify in toll-like receptor-2 (TLR-2) knockout (TLR2KO) and $\mu$-opioid receptor knockout (MORKO) animals whether morphine effects are influenced by these receptors. TLR2KO animals treated with placebo or morphine were compared with wild-type (WT) animals as above (Figure 2a). pCoA analysis of the five groups indicates WT-morphine as the only group that shows separation from the others, implying that TLR2KO-morphine animals are protected from the microbial shift.

Similar to TLR2KO profile, MORKO animals exhibited a pCoA distribution unperturbed by morphine (Figure $2 \mathbf{b}$ ). Although this was expected, one major difference seen here is a distinct clustering of the MORKO (both placebo- and morphine-implanted) animals away from the WT-placebo animals. This indicates that unconditional absence of $\mu$-opioid receptor has changed the basic composition of the microbiome in these animals, which is different from pharmacological inhibition of $\mu$-opioid signaling with naltrexone. Morphineinduced changes in the microbiome might be mediated by $\mu$-opioid signaling in the peripheral immune cells and could be because of an altered tutoring effect, by now a well-established phenomenon. ${ }^{17,24}$ To test this, we implanted placebo and morphine pellets into non-obese diabetic, severe-combined immune-deficient (NOD-SCID) with interleukin-2 (IL-2) receptor gamma knockout (NOD scid gamma (NSG); Jackson Laboratories) animals, with impaired innate and adaptive immune compartments. pCoA of NSG animals shows a very similar profile as MORKO animals, where there are fundamental differences in the basic microbial composition between WT and NSG, but morphine-mediated changes are completely abolished (Figure 2c). Additionally, bacterial translocation because of morphine treatment, a hallmark of gut barrier compromise, ${ }^{3,15}$ was significantly abrogated in the NSG-morphine 
a
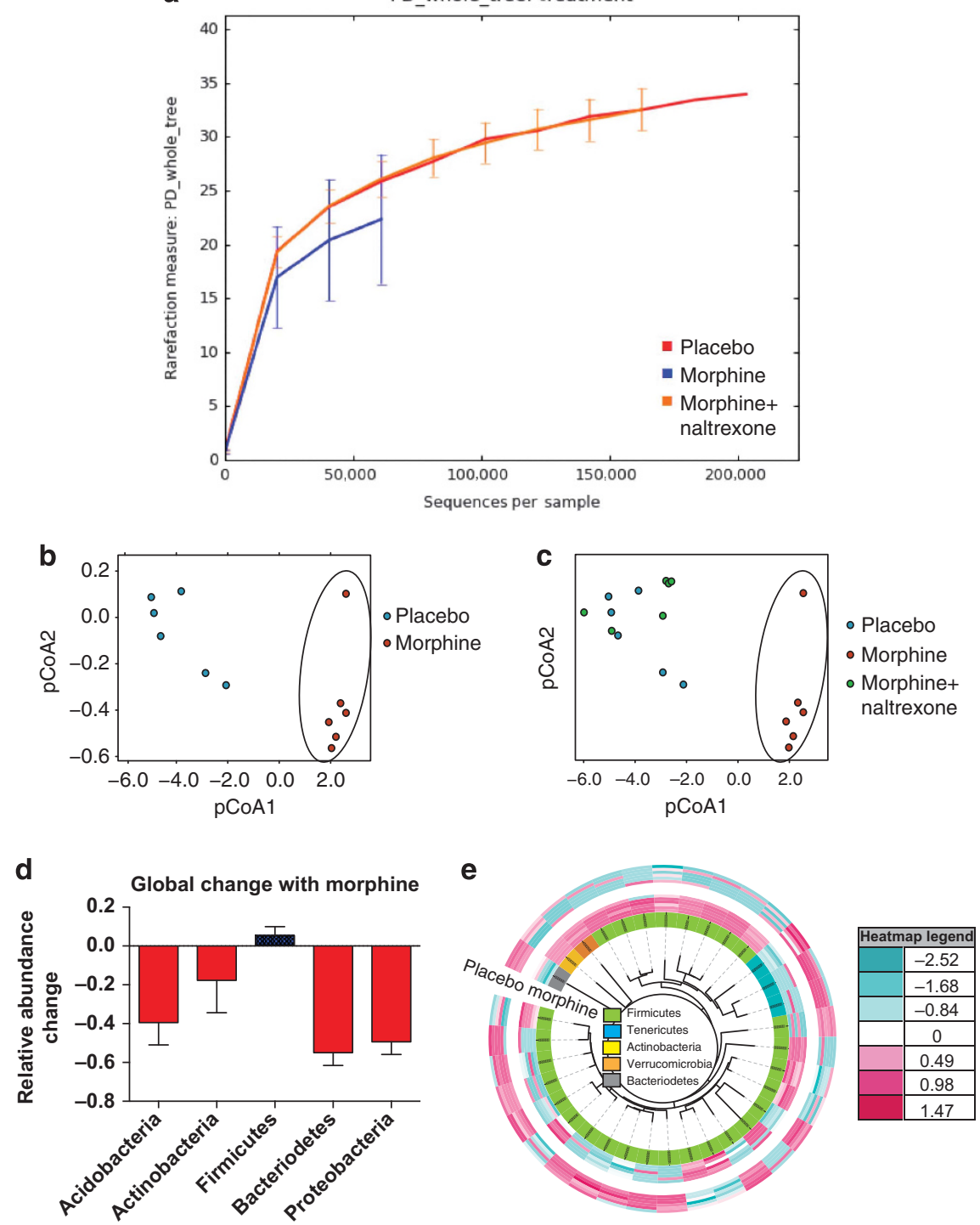

Figure 1 Morphine-induced global changes in the microbiome. (a) Phylogenetic diversity (PD) among the 16s rDNA content from animals treated with placebo, morphine, and morphine + naltrexone exhibits a trend (nonsignificant) towards reduced species richness for the morphine-treated animals. (b) Principal coordinate analysis ( $\mathrm{pCoA}$ ) of $16 \mathrm{~s}$ rDNA content from wild-type (WT) animals implanted with placebo or morphine (circled) pellets show distinct clustering of microbiome. (c) Microbial composition of animals coimplanted with morphine and naltrexone pellets show high similarity with placebo-implanted animals, distinct from morphine-implanted animals (circled). (d) Ratio of relative abundance of annotated operational taxonomic units OTUs between placebo- and morphine-implanted animals show a net expansion of phylum Firmicutes among five major commensal phyla. Top OTUs from each family, with significant difference between placebo and morphine groups, was rendered as a heat map using interactive tree of life (iToL; itol.embl.de). (e) In this heat map, 28 out of 34 OTUs belong to phylum Firmicutes with a net preferential expansion in the morphine-treated animals ( $n=6$ for pCoA analyses (see also Supplementary Figures S1 and S2)).

animals (Figure 2d). We have previously shown that bacterial translocation because of morphine is significantly diminished in the TLR2KO mice and completely abolished in the MORKO animals. ${ }^{3,15}$ The absence of morphine-mediated bacterial translocation and microbial changes in NSG animals strongly indicates a linear correlation between $\mu$-opioid receptor and TLR2 signaling in the mucosal immune cells influencing a focused change in the microbial composition of the animals. Inhibition/knockout of either of these receptors, irrespective of the baseline changes in the microbiome because of their absence, rescues the morphine-induced microbial dysbiosis and its physiological fallout with respect to bacterial translocation and gut barrier homeostasis.

\section{Microbial transplant influences commensal flora and gut morphology}

Fecal transplant has been successfully used clinically, especially for treating Clostridium difficile infection. ${ }^{25-28}$ With our expanding knowledge of the central role of microbiome in maintenance of host immune homeostasis, ${ }^{17}$ fecal transplant is gaining importance as a therapy for indications resulting from microbial dysbiosis. There is a major difference between fecal 
a

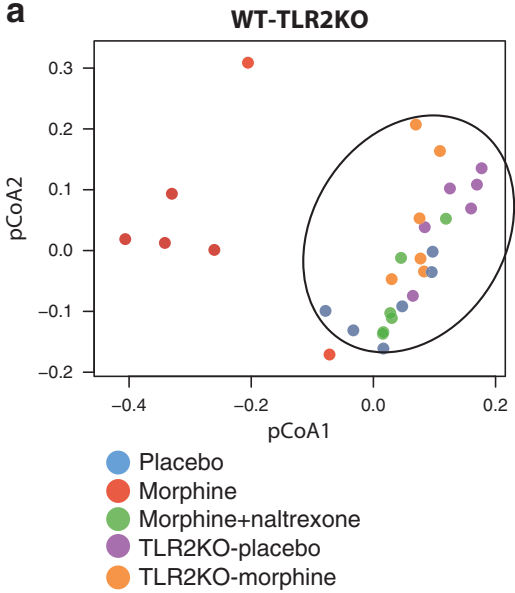

b

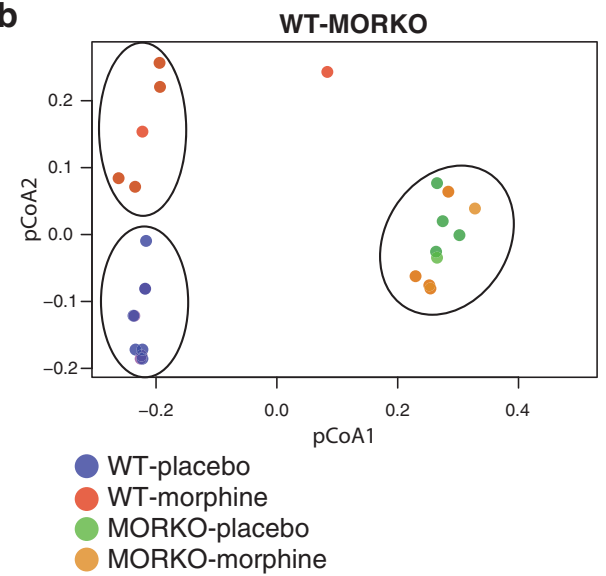

C

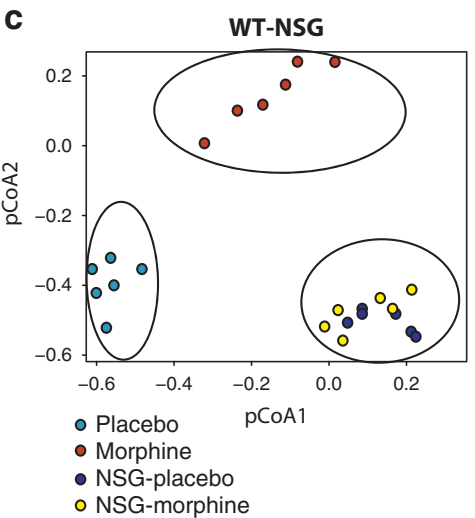

d

Bacterial translocation-liver

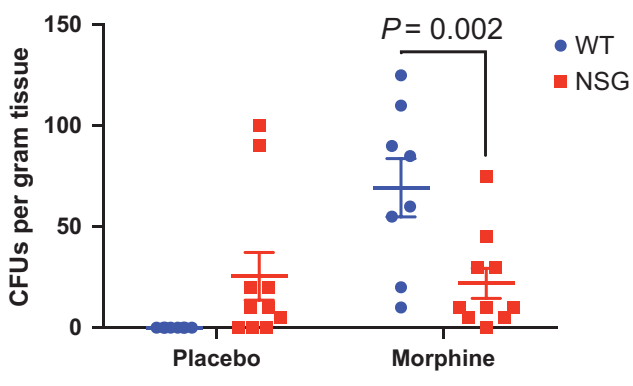

Figure 2 Dependence of morphine-induced microbial changes on toll-like receptor-2 (TLR2), $\mu$-opioid receptor, and host immune system. (a) TLR2KO animals, implanted with placebo or morphine pellets, exhibit similar microbial composition as placebo and morphine + naltrexone implanted wild-type (WT) animals in principal coordinate analysis ( $\mathrm{pCoA}$ ) analysis (circled), distinct from morphine-implanted WT animals. (b) $\mu$-Opioid receptor knockout (MORKO) animals, although exhibiting a distinct microbial composition compared with WT animals, do not show morphine-mediated microbial changes. (c) Similar to MORKO animals, severely immune-compromised non-obese diabetic scid gamma (NSG) animals exhibit a distinct microbial composition compared with WT animals, with no morphine-mediated changes in the microbiota. (Individual groups forming a distinct cluster circled in each pCoA plot. $N=6$ for each group.) Liver lysates of WT and NSG animals, implanted with placebo or morphine pellets for $72 \mathrm{~h}$ were plated on sheep-blood agar plates and total colony-forming units (CFUs) counted as a surrogate of gut barrier compromise and bacterial translocation. (d) While WT-morphine animals exhibited robust translocation of bacteria, NSG animals failed to exhibit morphine-mediated exacerbation in bacterial translocation ( $n=5$; lysate from each animal plated in duplicate).

transplant being used for the treatment of $C$. difficile infection and the conditions described in our studies. The former strategy is based on the argument that microbial dysbiosis caused by disproportionate overgrowth of a pathobiont can be outcompeted by reintroducing the missing flora by way of a "normal microbiome" transplant. This strategy is independent of host factors and systemic effects on the microbial composition. Here, we show that microbial dysbiosis due to morphine can be reversed by transplantation of microbiota from the placebo-treated animals. There is a distinct clustering of the transplanted microbiome (Figure 3a) and that "placebo to placebo (PP)" and "placebo to morphine (PM)" groups cluster together, which is distinct from "morphine to morphine (MM)" and "morphine to placebo (MP)" groups (Figure 3b). This indicates that the reciprocally transplanted microbiome tend to cluster together with the donor microbiome rather than the treatment condition. As an extension of the transplant experiment, we asked whether morphine-induced dysbiotic microbiome is capable of recapitulating gut pathologies in WT animals. Donor mice ( $48 \mathrm{~h}$ morphine) exhibit morphineinduced hallmark distortion of gut morphology as described earlier ${ }^{4}$ and early signs of morphological changes are evident in the recipient animals as well (Figure 3c). These data indicate that, if not all, a significant measure of gut pathology and resultant systemic inflammation is mediated by morphineinduced microbial dysbiosis, which can be partially restored with "normal" microbial transplant.

\section{Microbial transplant influences gut immunity}

Gut mucosal events leading to host inflammation follow a cascade, where resident macrophages and dendritic cells within the intestine carry the information to the mesenteric and gastric lymph nodes for lymphocyte priming. To study the effect of microbial dysbiosis and host immune response, we performed a multiplexed bead array (cytometric bead arrays) analysis of the mesenteric lymph node homogenate from the animals with 

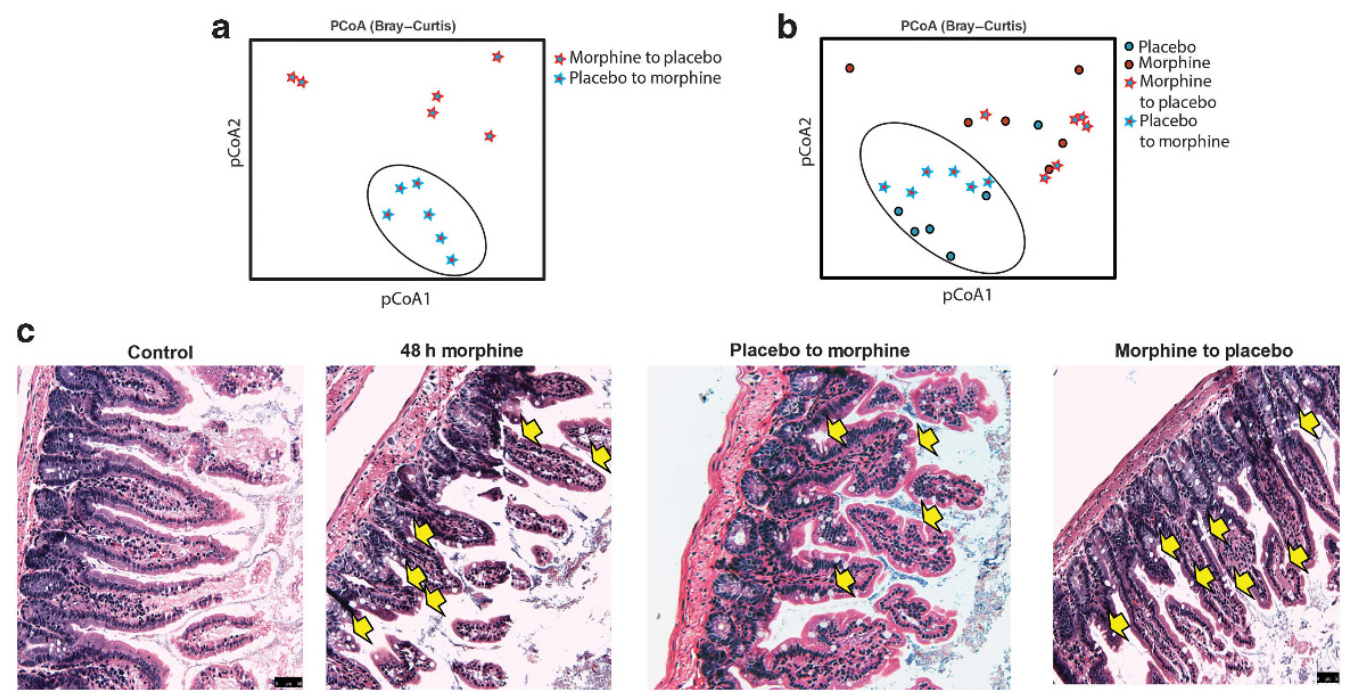

Figure 3 Donor microbiome predominates treatment condition and influences gut physiology. (a) Reciprocal transplant of microbiota preserves distinctness of microbial composition, which is closer to the donor microbiome, rather than the treatment condition (placebo or morphine alone; $\mathbf{b}$ ). (c) This physiologically translates to gut pathologies associated with morphine-induced dysbiotic microbiome, e.g., transplantation of morphine-induced dysbiotic microbiome into healthy wild-type (WT) animals results in "morphine-like" diseased phenotype, very similar to morphine-implanted donor animals, whereas transplantation of "normal" microbiome into morphine-treated animals shows distinct improvement in the gut pathology. Arrows indicate sites of gut injury.

a

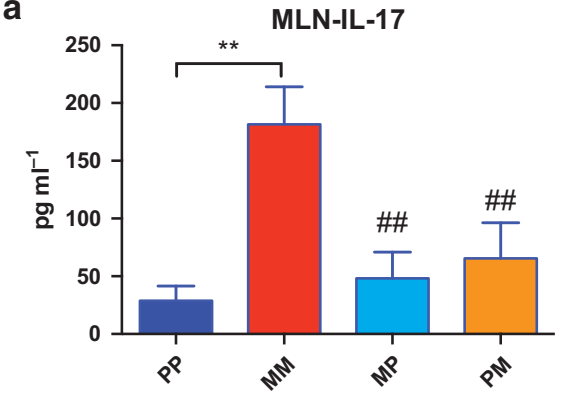

C

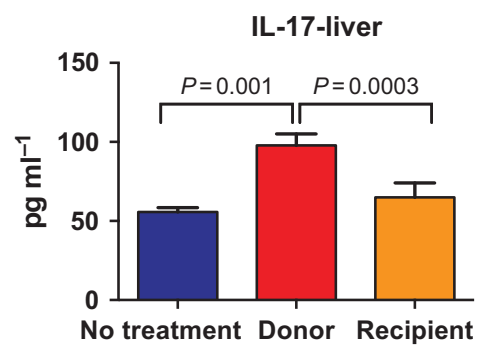

b

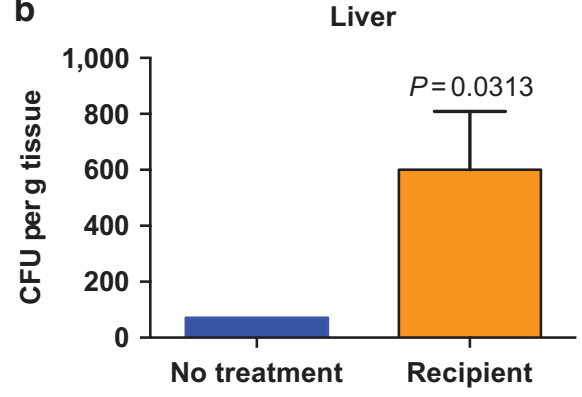

d

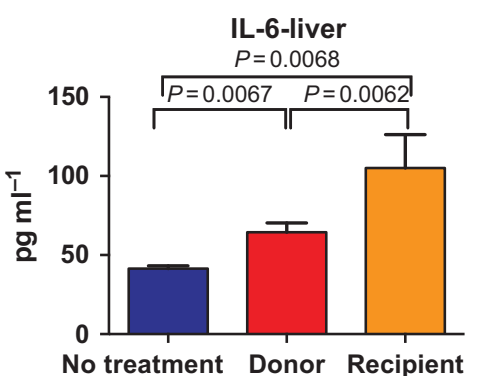

Figure 4 Microbial transplant, immune response, and rescue. (a) Morphine treatment results in a robust interleukin-17 (IL-17) response in the mesenteric lymph node (MLN) and transplantation of placebo microbiome rescues the inflammatory phenotype. (b) Reconstitution of dysbiotic microbiome alone is sufficient to recapitulate most of the morphine-induced gut pathologies, including bacterial translocation across the intestinal mucosa. Systemic IL-17 response was only seen in (c) donor animals with morphine treatment and (d) both donors and recipients exhibit a systemic IL-6 response indicating proinflammatory environment in the host. In all cases (a-d), morphine-implanted animals receiving "normal" microbiota show baseline levels of IL-17 ( ${ }^{\star \star} P<0.05$ between indicated groups; ${ }^{\# \#} P<0.05$ compared with morphine to morphine (MM)). Also see Supplementary Figure S3.

fecal transplant from above. Among all cytokines analyzed, only IL-17 and IL-10 showed significant differences among groups. Elevated levels of IL-17 (Figure 4a) and attenuated levels of IL-10 (Supplementary Figure S3A) were seen in MM compared with the rest of the four study groups, consistent with the described role of IL-17 in host inflammation arising from compromised gut homeostasis, specifically in the context of Gram-positive infection. ${ }^{29-32}$ The reciprocally transplanted 
groups, MP and PM, maintained basal levels of the cytokines, comparable with PP. The lack of IL-17 or IL-10 response in MP or PM animals could be because of compensatory (averaging) effects between microbiome and host immune system. Alternatively, in MP animals, transplanting dysbiotic microbiome may change the general composition of the commensal flora and yet may not have resulted in severe mucosal barrier compromise to affect systemic immune response within $72 \mathrm{~h}$. In PM animals, on the other hand, some measure of rescue because of microbial restoration may have resulted in diminished proinflammatory response.

Additionally, we set out to determine the overall systemic response to microbial dysbiosis by investigating the blood cytokine levels in these animals. No significant differences were found among the WT, TLR2KO, and MORKO animals under different treatments (Supplementary Figure S3B-D). A significant decrease in IL-2 and IL-4 levels was observed in the reciprocal fecal transplant animals compared with PP and MM, but no corresponding elevation in proinflammatory cytokines was observed (Supplementary Figure S3E). This indicates that the $72 \mathrm{~h}$ time point may not be sufficient to elicit proinflammatory biomarkers in the systemic circulation; however, localized inflammatory response is initiated within the intestine and mesenteric drainage in immunocompetent animals. We have previously shown that morphine exacerbates TLR-mediated inflammation, ${ }^{3,4,15}$ and basal levels of inflammation in the PM animals clearly exhibits protective effect because of the restoration of microbial homeostasis with fecal transplant.

In the absence of systemic morphine, fecal transplant of dysbiotic microbiome alone was sufficient to induce bacterial translocation to the liver (Figure 4b). Similar to MM animals in Figure 4a, fecal transplant induced IL-17 response in the liver of the donor mice, but not in the recipient animals (Figure 4c). There was a significant IL-6 response both in donor and recipient animals (Figure 4d), indicating that IL-17-mediated gut inflammation is contingent upon morphine-mediated host immunomodulation, whereas IL-6-mediated systemic inflammation is a result of bacterial translocation, common to both donors and recipient animals. This is consistent with our observation that IL-17 is an early response, whereas IL-6 is the sustained host response to morphine-mediated changes in gut homeostasis. ${ }^{4,12}$

\section{Morphine-induced cholesterol/bile acid imbalance}

Recent reports implicate increase in hydrophobic secondary bile acids (lithocholic acid and deoxycholic acid) and concomitant decrease in hydrophilic secondary bile acids (ursodeoxycholic acid (UDCA)) in gut barrier disruption, bacterial translocation, and intestinal inflammation. ${ }^{13,14}$ As secondary bile acids are produced in entirety through gut microbial fermentation from primary bile acids (cholic acid and chenodeoxycholic acid), secondary bile imbalance is directly correlated, and associated with microbial dysbiosis. ${ }^{33}$ Exclusively within the host liver, primary bile acids are produced from cholesterol catabolism and is, in fact, one of the major ways of eliminating excess cholesterol from the body. ${ }^{34}$ The secondary bile acids recirculate to the host liver through various bile transporters and also signal through intestinal receptors, constituting the hepatoenteric bile circulation. ${ }^{11,35}$

To evaluate if morphine treatment induces metabolic changes in the gut, WT and TLR2KO animals were implanted with placebo or morphine pellet for $72 \mathrm{~h}$ as described above and the fecal content was analyzed with mass spectrometry for changes in major metabolites. Of 310 compounds tested, lipid metabolites and especially bile acids (both primary and secondary) exhibited significant changes because of morphine (Supplementary Figure S4). One of the major observations was significantly high level of coprostanol in the morphineimplanted animals (Figure 5a). This could be because of abnormal release of cholesterol into the gut or because of selective expansion of microbes known for this conversion. On the other hand, concentrations of the host-derived primary (Figure $5 \mathbf{b}, \mathbf{c}$ ) and microbe-converted secondary bile acids (Figure 5d,e) were seen to diminish significantly in the feces of morphine-implanted animals compared with placebo and morphine + naltrexone-implanted animals. The morphinemediated changes were completely abolished in the TLR2KO animals (Figure 5f).

To understand the metabolic status of the host in the context of hepatoenteric circulation and bile metabolism, we subjected the liver lysate of the animals to mass spectrometric analysis. A significantly elevated level of cholesterol was seen in morphineimplanted mice (Figure 5g). These effects are abolished in MORKO animals and with naltrexone. No significant differences were observed between placebo- and morphineimplanted TLR2KO mice; however, the basal cholesterol levels in these animals were significantly lower compared with their WT counterparts. Primary bile acid (CA) did not show any significant difference in the levels because of morphine treatment (Supplementary Figure S5A) and neither did secondary bile acids (deoxycholic acid, taurocholic acid, and lithocholic acid; Supplementary Figure S5B-D). Unconjugated UDCA, on the other hand, was seen to be significantly downregulated in the morphine-implanted animals (Figure $\mathbf{5 h}$ ). As this is a secondary bile acid, its presence in the host liver can only be explained by biliary reabsorption from the intestine. One of the major bacterial enzymes involved in the deconjugation of secondary bile acids is the bile salt hydrolase, and its activity determines the efficacy of hepatoenteric circulation. ${ }^{33,36,37} \mathrm{We}$ observed a significant reduction of bile salt hydrolase activity within the gut bacteria because of morphine treatment (Figure 5i) and significantly reduced free taurine in the feces with mass spectrometry (Figure 5j). As a direct correlation, among gut bacteria implicated in the deconjugation of bile acids, ${ }^{38,39}$ we observed significant morphine-mediated lowering in relative abundance of OTUs representing Lactobacillus and Clostridium (Figure 6). While morphine treatment promotes the expansion of pathogenic/translocating bacteria ${ }^{23}$ (and Supplementary Figure S1), it reduces specific bacteria that are responsible for the maintenance of metabolic homeostasis in the gut. Decrease in these bacterial communities may 
a

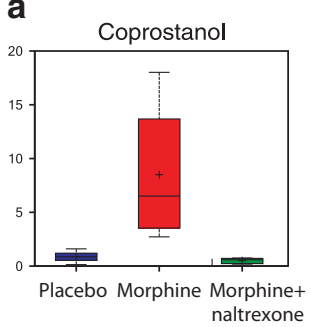

b

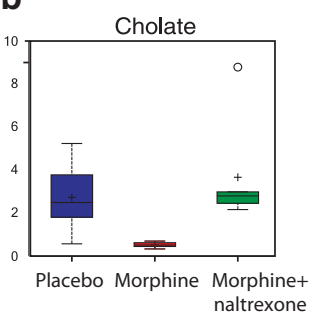

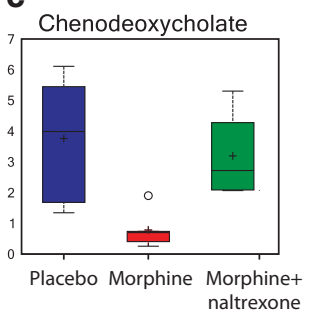

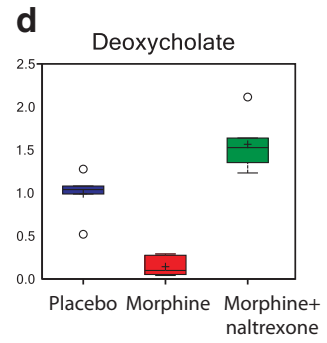

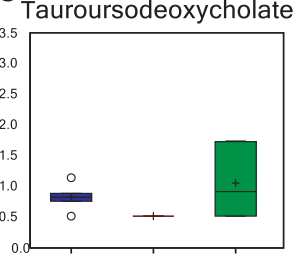

Placebo Morphine Morphine+ f

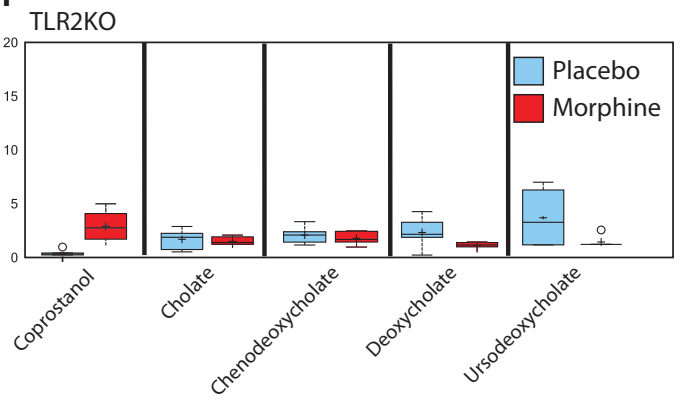

9

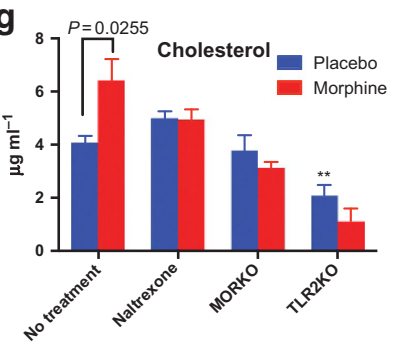

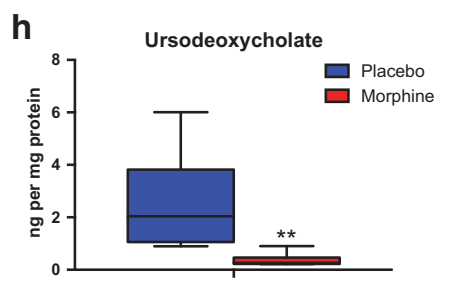

i

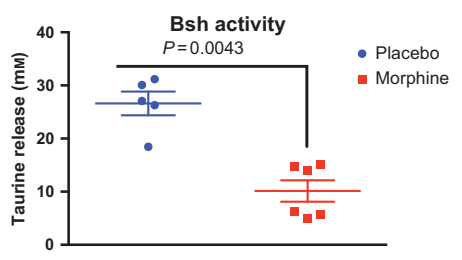

j

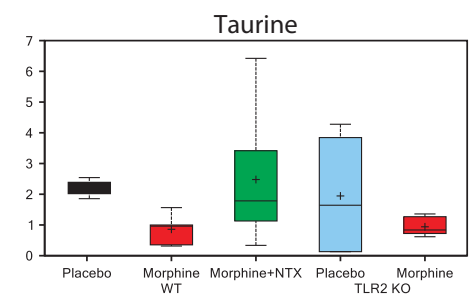

Figure 5 Morphine-induced intestinal and hepatic metabolic changes. (a) One of the major observations from these studies was that morphine treatment induces the production of significantly high amounts of coprostanol, a direct microbial conversion product of cholesterol in the intestine. There is a significant reduction in the abundance of primary bile acids, (b) CA and (c) chenodeoxycholic acid(CDCA) and (d) secondary bile acids deoxycholic acid (DCA) and (e) ursodeoxycholic acid (UDCA) in the fecal contents of morphine-treated mice. These affects are reversed in (a-e) morphine + naltrexone (NTX) treated and (f) TLR2KO animals. (g) Morphine induces significant accumulation of cholesterol in the liver, an effect, not seen in naltrexone-treated, $\mu$-opioid receptor knockout (MORKO) and TLR2KO animals. (h) These changes in bile/cholesterol levels ultimately result in disrupted hepatoenteric circulation as shown by reduced recovery of UDCA in the liver. (i) Bile salt hydrolase (Bsh), one of the major bacterial enzymes, important for an efficient hepatoenteric circulation shows significantly reduced activity in the microbiome of morphine-treated animals. (j) Hence, as expected, a lower level of free taurine was observed in the fecal content of morphine-treated animals, with no significant changes in the TLR2KO animals. Also See Supplementary Figures S4 and S5. KO, knockout; TLR, toll-like receptor; WT, wild type. 'o' denotes extreme data point, ' + ' denotes statistical mean. ** $p<0.05$ compared to TLR2KO-morphine group.

a

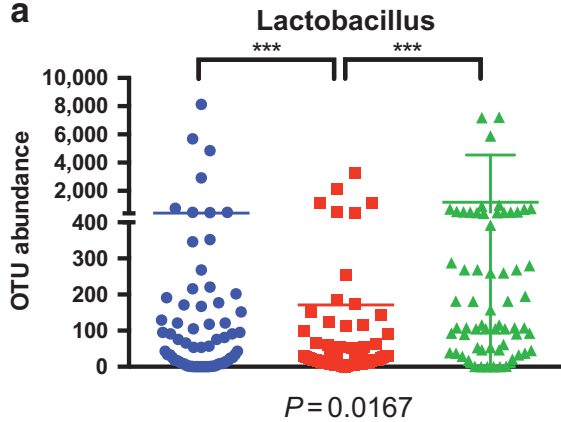

b

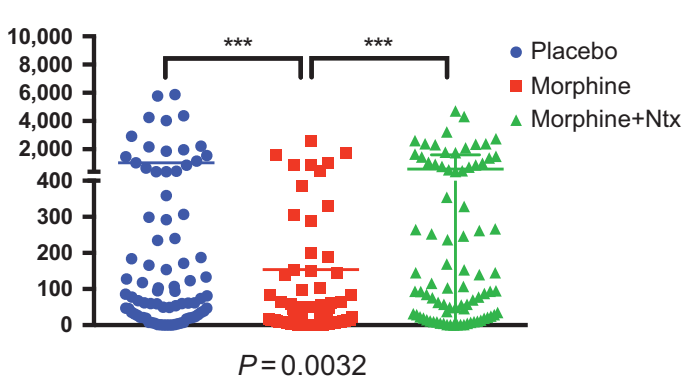

Figure 6 We observed a significant reduction in the abundance of OTUs associated with the genera (a) Lactobacillus and (b) Clostridium in morphine-treated mice. These genera have been strongly implicated in deconjugation of secondary bile acids, enabling their reabsorption through

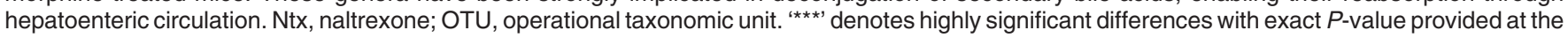
bottom of each panel.

explain the increased cholesterol levels in the liver of morphineimplanted animals because of disruption in hepatoenteric circulation of deconjugated bile acids and rate-limiting steps in cholesterol catabolism. In MORKO and TLR2KO animals (Supplementary Figure S5E and F), the levels of secondary bile acids, including UDCA, were similar between placebo- and 
morphine-implanted mice. CA, the primary bile acid, was significantly diminished in the morphine-implanted TLR2KO animals. This implicates the role of TLR2 in the morphinemediated modulation of cholesterol hydroxylation in the liver.

\section{DISCUSSION}

Currently, there is no alternative to morphine (and its derivatives) for efficient pain management in medical practice. Off-target effects of morphine, especially on the peripheral immune system, however, remain a concern in several disease conditions. ${ }^{3,5}$ Dysbiotic microbiome has been independently shown to promote a constant state of inflammation, which becomes critical in the management of several maladies including cancer and HIV infection, confounding the treatment process. ${ }^{17}$ Additionally, microbial dysbiosis is also correlated with gut barrier compromise and bacterial translocation, leading to increased inflammation and endotoxemia. ${ }^{23,39,40}$ One of the major physiological consequences of opioid use is severe constipation, which is speculatively implicated for morphine-induced gut pathologies including barrier compromise and bacterial translocation. In our studies, we have seen that constipation resulting from non-opioid inducers, e.g., low fiber diet, does not result in gut barrier disruption/bacterial translocation (Supplementary Figure S6). In various disease models describing morphine-mediated comorbidities, gut barrier compromise, bacterial translocation, and uncontrolled inflammation has a dominant role. ${ }^{4,19,20}$ Here, we have shown a distinct Gram-positive skew in the microbial composition following morphine treatment, which strongly correlates to the clinical presentations attributed to relative increase of Gram-positive phyla within the microbiota. ${ }^{23,41}$ While gut commensal flora constitutes a complex ecosystem, stress and disease in the host allows for certain simplified, yet strongly indicative changes in the microbial composition. The Firmicutes-Bacteroidetes ratio is one of those "markers" of proinflammatory changes in the microbiome; so far, studies have been conducted mostly in the context of aging, obesity, and diabetes. ${ }^{9,16}$ In this study, we demonstrate a skew of this ratio towards a proinflammatory phenotype, well corroborated with the host immune status involving innate responses. The role of TLRs is well established in mucosal pathogenic complications. Both TLR2 and 4 have a major role in morphine-mediated gut barrier compromise and inflammation. ${ }^{4,24,40,42-44}$ Hence, as shown here, morphineinduced microbial dysbiosis and inflammation is affected through gut barrier compromise and commensal bacterial translocation through gut mucosa, in a TLR2- and $\mu$-opioid receptor-dependent manner. ${ }^{3,40}$ We have recently shown ${ }^{3,23}$ that morphine treatment results in gut barrier compromise and translocation of predominantly, Gram-positive bacteria across gut mucosa. We have also shown ${ }^{3}$ that TLR stimulation results in myosin light-chain kinase-mediated withdrawal of tightjunction proteins from the gut epithelial membrane and resultant barrier compromise. In this manuscript, we show the essential role of host immune system in morphine's effect on microbial dysbiosis (no effect of morphine on microbiome in immunocompromised NSG animals; Figure 2c), implying that morphine's effects on the microbiome is routed via immune modulation. Finally, we show here that TLR2KO animals, similar to NSG, do not show microbial dysbiosis, implicating the role of TLR2 in morphine-mediated immune changes, resulting in microbial dysbiosis. One of the functional consequences of morphine-induced microbial dysbiosis is the reprogramming of the host immune status. ${ }^{17,24,39-41,45,46} \mathrm{In}$ this study, we clearly show that morphine-induced dysbiotic microbiome alone can recapitulate diseased gut pathology and immune responses and it is possible to reverse microbial dysbiosis and restore gut immune homeostasis with fecal transplant, which has immense therapeutic potential, as shown previously for treating C. difficile infection. ${ }^{25-28}$ The second and more direct physiological effect of morphine-mediated microbial dysbiosis is its consequence on hepatoenteric circulation of bile acids. Recent reports indicate that bile acid metabolism, its pool in the host, and release into the gut has a significant role in the manifestation of gut barrier pathology and resultant inflammation. Recently, modulation of cholesterol- $7 \alpha$-hydroxylase in the liver and farnesoid-x-receptor in the liver and intestine have been implicated in bile acid dysbiosis and gut barrier compromise. ${ }^{13,14,47,48}$ In this study, morphine induced accumulation of cholesterol in the liver and its excessive conversion to coprostanol in the intestine. Primary and secondary bile acids, however, decreased in the intestine, indicating morphine-induced altered cholesterol metabolism in the liver and intestine. At the same time, altered bile release in the gut has adverse consequences in the expansion and maintenance of specific bacterial communities, where bile acids and their conjugation status influence both sporulation and germination process. ${ }^{12,33,49}$

Our results clearly show a linear correlation between morphine-mediated microbial dysbiosis, disruption of cholesterol/bile acid metabolism, and barrier disruption, promoting sustained inflammation in the host, although the sequence of events are still not clear. We have also demonstrated that microbial reconstitution and timely blockade of TLR2/MOR signaling can restore gut homeostasis in morphine-implanted animals. Additional studies are required to understand the temporal relationship between morphine treatment, bile acid imbalance, microbial dysbiosis, and role and status of bile regulatory receptors, e.g., farnesoid-x-receptor, ${ }^{50}$ bile transporters, and the feedback loop including cholesterol- $7 \alpha$ hydroxylase ${ }^{35}$ in the host liver to effectively exploit microbial and bile acid modulation as secondary therapeutic strategy on patients maintained on morphine for pain management.

\section{METHODS}

Materials and reagents. Antibodies for flow cytometry were purchased from BD Biosciences (San Jose, CA). Cytokine levels were determined using 13-plex Cytometric Bead Arrays from BioLegend (San Diego, CA). Mass spectrometry reagents were sourced from various vendors as follows: JT Baker Ultra liquid chromatographymass spectrometry-grade acetonitrile, methanol, and water were purchased from VWR International (Radnor, PA). Liquid chromatography-mass spectrometry ultra-grade formic acid (Fluka) was 
purchased from Sigma-Aldrich (Saint Louis, MO). Internal standards 2,2,4,4- $\mathrm{D}_{4}$ cholic acid, 2,2,4,4- $\mathrm{D}_{4}$ deoxycholic acid, 2,2,4,4- $\mathrm{D}_{4}$ chenodeoxycholic acid, 2,2,4,4- $\mathrm{D}_{4}$ ursodeoxycholic acid, and 2,2,4,4- $\mathrm{D}_{4}$ lithocholic acid were purchased from Cambridge Isotope Laboratories (Andover, MA). 2,2,4,4- $\mathrm{D}_{4}$ taurocholic acid was purchased from AlsaChim (Illkirch-Graffenstaden, France). Millipore Amicon Ultrafree PTFE membrane centrifugal filters $(0.2 \mu \mathrm{m})$ and Millipore Amicon Ultra $0.5 \mathrm{ml} 3,000 \mathrm{MWCO}$ centrifugal filters were purchased from Thermo Fisher Scientific (Waltham, MA).

Mice. C57BL/6 and NSG mice were purchased from Jackson Laboratories (Bar Harbor, ME). TLR2KO and MORKO mice were bred in-house. All animals were maintained in pathogen-free facilities and all procedures were approved by the University of Minnesota Institutional Animal Care and Use Committee. Typically, 8-10-weekold animals were used for our studies.

Placebo/morphine/naltrexone pellet implantation. Slow release morphine pellets $(25 \mathrm{mg} ; \sim 1 \mu \mathrm{M}$ serum levels of morphine for 5-6 days) and corresponding placebo or naltrexone pellets, as appropriate, were kindly provided by National Institute of Drug Abuse (NIDA, National Institutes of Health, Rockville, MD). The implantation procedure involved 3\% isoflurane-induced anesthesia, followed by making a small incision at the dorsal torso of the mice. The appropriate pellet was inserted into the small pocket created during incision and the wound was closed using stainless-steel wound clips. The whole process was carried out under aseptic conditions.

Fecal transplant. Two batches of C57Bl6/j (WT) animals (10 each) were implanted with placebo or morphine pellets as mentioned above for 24 or $48 \mathrm{~h}$ and their fecal contents collected and pooled. The fecal content was processed according to fecal microbial transplant procedure described for human patients ${ }^{51}$ with modifications. Briefly, the fecal contents were suspended in phosphate-buffered saline $\left(10 \mathrm{mg} \mathrm{ml}^{-1} ; \mathrm{w} \mathrm{v}^{-1}\right)$, filtered through a $40 \mu$ mesh and centrifuged at $6,000 \mathrm{~g}$ for $20 \mathrm{~min}$. The resultant microbial pellet was resuspended in half volume of chilled phosphate-buffered saline and aliquoted into volumes for single thaw and use. Aliquotes destined for later use were reconstituted to $10 \%$ sterile glycerol (Sigma, St. Louis, MO) and stored at $-80^{\circ} \mathrm{C}$. A total of $32 \mathrm{WT}$ mice were used for the transplant experiment. Animals were implanted with placebo or morphine pellets (12 each) and the stored microbiota was administered $\left(10^{6}\right.$ colonyforming units (CFUs) per dose) via oral gavage every $24 \mathrm{~h}$ three times according to the following scheme: (a) placebo pellet-implanted animals getting placebo microbiome (PP; $n=8)$, (b) placebo pellet-implanted animals getting morphine microbiome (PM; $n=8$ ), (c) morphine pellet-implanted animals getting placebo microbiome (MP; $n=8$ ), and (d) morphine pellet-implanted animals getting morphine microbiome (MM; $n=8)$. Animals were killed $24 \mathrm{~h}$ after third transplant and fecal contents and tissues harvested for various downstream analyses as described.

Sequencing and 16S DNA analysis. Fecal content was collected from the gut region encompassing distal cecum and $\sim 1$ in of the colon and frozen on dry ice. The fecal matter was lysed using glass beads in MagnaLyser tissue disruptor (Roche, Indianapolis, IA) and total DNA was isolated using Power-soil/Fecal DNA Isolation Kit (Mo-Bio, Carlsbad, CA) as per the manufacturer's specifications. All samples was quantified via the Qubit Quant-iT dsDNA Broad-Range Kit (Invitrogen, Life Technologies, Grand Island, NY) to ensure that they met minimum concentration and mass of DNA and submitted to either Second Genome (San Fransisco, CA) or University of Minnesota Genomic Center (Minnesota, MN) for microbiome analysis as follows: to enrich the sample for the bacterial $16 \mathrm{~S}$ V4 rDNA region, DNA was amplified using fusion primers designed against the surrounding conserved regions that are tailed with sequences to incorporate Illumina (San Diego, CA) flow cell adapters and indexing barcodes. Each sample was PCR amplified with two differently barcoded V4 fusion primers and were advanced for pooling and sequencing. For each sample, amplified products were concentrated using a solid-phase reversible immobilization method for the purification of PCR products and quantified by electrophoresis using an Agilent (Santa Clara, CA) 2100 Bioanalyzer. The pooled 16S V4-enriched, amplified, barcoded samples were loaded into the MiSeq cartridge (Illumina Inc, San Diego, $\mathrm{CA}$ ) reagent cartridge, and then onto the instrument along with the flow cell. After cluster formation on the MiSeq Instrument (Illumina, San Diego, CA), the amplicons were sequenced for 250 cycles with custom primers designed for paired-end sequencing. Using QIIME, sequences were quality filtered and demultiplexed using exact matches to the supplied DNA barcodes. The resulting sequences were then searched against the Greengenes reference database of $16 \mathrm{~S}$ sequences, and clustered at $97 \%$ by uclust (closed-reference OTU picking). Analysis for $\alpha$ - and $\beta$-diversity was carried out with standardized qiime workflow at the Minnesota Supercomputing Institute (University of Minnesota, Minneapolis, MN). To compute the "global changes with morphine" histogram (Figure 1d), relative abundance for all OTUs constituting a single phylum in morphine-treated animals were normalized with the same OTUs in the placebo animals, including mismatched OTUs. The resulting ratio was analyzed using Prism (GraphPad, LaJolla, CA) software to understand morphine-mediated perturbations in the five major phyla within the microbiome. The raw data files for $16 \mathrm{~s}$ rDNA sequencing have been deposited with ArrayExpress with the accession numbers E-MTAB-3722 (native effects of morphine) and E-MTAB-3723 (microbial transplant).

Mass spectrometry for gut and liver metabolites. Fecal metabolites were analyzed by Metabolon (Research Triangle Park, NC) using their proprietary 310 named biochemicals screen. Based on the results, independent analysis of liver bile acids was performed at the University of Minnesota mass spectrometric facility as follows: samples for analysis by ultra-performance liquid chromatography-tandem mass spectrometry (MS/MS) were spiked with a fixed volume of the isotopically labeled bile acid internal standards described above. To each sample was added an equal volume of liquid chromatographymass spectrometry-grade methanol. The samples were then centrifuged at 12,000 r.p.m. for $5 \mathrm{~min}$ and the supernatants removed from the proteinaceous pellet. These supernatants were centrifuged through an Amicon Ultrafree low-binding hydrophilic polytetrafluoroethylene membrane $(0.2 \mu \mathrm{m})$ at 12,000 r.p.m. and the filtrates collected. These filtrates were then centrifuged at $14,000 \mathrm{~g}$ through an Amicon Ultra $3 \mathrm{kDa}$ molecular weight cutoff filtration column (Millipore, Billerica, $\mathrm{MA}$ ) for $30 \mathrm{~min}$, and the flow-through collected for ultra-performance liquid chromatography-MS/MS analysis. A Waters Acquity UPLC coupled to a Waters triple quadrupole mass spectrometer (Acquity TQD, Waters Corporation, Milford, MA) was used for separation and detection of bile acids. A Waters CORTECS $\mathrm{C}_{18} 2.1 \mathrm{~mm} \times 100 \mathrm{~mm}$ column $(2.7 \mu \mathrm{m}$ particles $)$ at $40{ }^{\circ} \mathrm{C}$ was used during the following 19 min gradient separation with A: water containing $0.1 \%$ formic acid and $\mathrm{B}$ : acetonitrile containing $0.1 \%$ formic acid, at a flow rate of $0.6 \mathrm{ml} \mathrm{min}^{-1}$ : $35-40 \%$ B, 0-1.5 min; $40-50 \%$ B, $1.5-6.0 \mathrm{~min} ; 50 \% \mathrm{~B}$ 6.0-7.0 min; 50-97\% B, 7.0-14.0 min; 97\% B, 14.0-16.0 min; 97-35\% $\mathrm{B}, 16.0-17.0 \mathrm{~min}$; and $35 \% \mathrm{~B}, 17.0-19.0 \mathrm{~min}$. By directly infusing each of the bile acids and corresponding internal standards, cone voltages and collision energies for each selected reaction monitoring transition were optimized. The transitions that produced the highest sensitivity for the determination of each analyte were selected for quantification. Note that MS1 selected ion monitoring of the precursor ions specified lacked the sensitivity and selectivity of MS2 measurement of the following precursor-to-precursor MS/MS transitions. Note also that the method of development and validation included identity verification via unique precursor-fragment MS/MS transitions for each analyte. The following transitions were selected for quantitative analysis: lithocholic acid: $375.4-375.4 ; 2,2,4,4-\mathrm{D}_{4}$ lithocholic acid: 379.4-379.4; cheno-urso- and deoxycholic acids: 391.4-391.4; 2,2,4,4- $\mathrm{D}_{4}$ cheno-urso- and deoxycholic acids: 395.4-395.4; cholic acid: 407.3-407.3; 
2,2,4,4- $\mathrm{D}_{4}$ cholic acid: 411.3-411.3; taurocholic acid: 514.1-514.1; 2,2,4,4- $\mathrm{D}_{4}$ taurocholic acid: 518.1-518.1. Dwell time for each transition was $0.05 \mathrm{~s}$. For electrospray ionization-MS/MS in negative ionization mode, parameters were as follows: capillary, $3.2 \mathrm{kV}$; cone, $70 \mathrm{~V}$; extractor, $3 \mathrm{~V}$; rf lens, $0.3 \mathrm{~V}$; source temperature, $150{ }^{\circ} \mathrm{C}$; desolvation temperature, $500^{\circ} \mathrm{C}$; desolvation flow, $800 \mathrm{lh}^{-1}$; cone gas flow, $20 \mathrm{lh}^{-1}$; low-mass resolution (Q1), $15 \mathrm{~V}$; high-mass resolution (Q1), $15 \mathrm{~V}$; ion energy $(\mathrm{Q} 1), 0.2 \mathrm{~V}$; entrance, $-5 \mathrm{~V}$; exit, $1 \mathrm{~V}$; collision energy, $5 \mathrm{~V}$; low-mass resolution $(\mathrm{Q} 2), 15 \mathrm{~V}$; high-mass resolution (Q2), $15 \mathrm{~V}$; and ion energy (Q2), 3.5 V. For standardization, six levels of calibration mixtures for each bile acid ranging from 0 to $250 \mu \mathrm{g} \mathrm{ml}^{-1}$ were prepared to achieve six different response ratios in the mixtures. These solutions were then analyzed by UPLC-MS/MS, and the data were subjected to a linear least-squares analysis with the Waters Targetlynx software program (Waters Corporation). The peak area ratios of analyte:internal standard were then used in conjunction with the calibration curves to determine the concentrations of bile acids in the samples.

Bile salt hydrolase assay. For microbial bile salt hydrolase assay, method described in Kumar et al. ${ }^{37}$ was used with modifications. Briefly, weight-matched fecal content from placebo and morphinetreated animals were resuspended in chilled phosphate-buffered saline and filtered sequentially through 100, 40, and $20 \mu$ mesh (BD Biosciences). The filtered suspension was centrifuged at $500 \mathrm{~g}$ (supernatant collected), $1000 \mathrm{~g}$ (supernatant collected), and, finally, $10,000 \mathrm{~g}$ (pellet collected). The microbial pellets were resuspended in $100 \mu \mathrm{l}$ of $0.5 \mathrm{M}$ citrate buffer and bacterial cells were disrupted using an ultrasonic homogenizer. Bacterial cytoplasmic content was separated from debris by centrifugation $(20,000 \mathrm{~g})$ and the supernatant was used for bile salt hydrolase assay. Bacterial cytoplasmic fraction was incubated with or without $0.5 \mathrm{~m}$ tauro-deoxycholate (Fisher) at $37^{\circ} \mathrm{C}$ for $30 \mathrm{~min}$ and release of free taurine was measured using $1 \%$ ninhydrin at $570 \mathrm{~nm}$. Bacterial cytoplasmic fraction, without taurodeoxycholate incubation was used to determine free amino acids and used as a background for normalization.

Histology. Tissues were harvested and preserved in $10 \%$ formaldehyde. Hematoxylin and eosin staining was performed by the Comparative Pathology Shared Resource (CPSR and Bionet) at the University of Minnesota and slides were imaged using a Leica DM5500 B microscope (Leica biosystems, Buffalo Grove, IL). Representative images are shown.

Statistical analysis. Microbiome analysis: OTU tables were rarefied to the sample containing the lowest number of sequences in each analysis. Qiime 1.8 was used to calculate $\alpha$-diversity (alpha_rarefaction.py) and to summarize taxa (summarize_taxa_through_plots.py). pCoA was carried out within this program using observation ID level. Heatmaps were generated using family level (L5) taxonomic data using R-based Phyloseq or using Explicet as described. The Adonis test was used for finding significant whole microbiome differences among discrete categorical or continuous variables. In this randomization/Monte Carlo permutation test, the samples were randomly reassigned to the various sample categories, and the mean normalized cross-category differences from each permutation are compared with the true crosscategory differences. The fraction of permutations with greater distinction among categories (larger cross-category differences) compared with that observed with the non-permuted data reported as the $P$-value for the Adonis test. Cytokine concentrations and bile acid changes from the plasma and liver is expressed as \pm s.e.m. Significance is defined as $P<0.05$ between groups in an unpaired Student's $t$-test. Bacterial counts were reported as means of CFU and were analyzed by the Mann-Whitney $U$-test (GraphPad Prism). For metabolite analysis by mass spectrometry, Welch's two-sample $t$-test was used to identify biochemicals that differed significantly between experimental groups. An estimate of the false discovery rate $(q$-value $<0.10)$ was calculated to take into account the multiple comparisons that normally occur in metabolomic-based studies. Biochemical importance plot was obtained using random forest analysis (a statistical tool for biomarker selection using a supervised classification technique based on an ensemble of decision trees). In this study, the metabolic profiles of fecal samples from five groups (WT + placebo, $\mathrm{WT}+$ morphine, $\mathrm{WT}+$ morphine + NTX, TLR2KO + placebo, and TLR2KO + morphine) were compared among each other. Supplementary Figure S4A lists the top 30 candidates based on importance to separating genotype/ treatment. A predictive accuracy of $97 \%$ was observed based on key differences in lipid and bile metabolism.

SUPPLEMENTARY MATERIAL is linked to the online version of the paper at http://www.nature.com/mi

\section{ACKNOWLEDGMENTS}

This work was supported, in part, by the NIH grants RO1 DA 12104, RO1 DA 022935, RO1 DA031202, K05DA033881, P50 DA 011806, 1R01DA034582, 1R21HL125021 (to SB), and 1R01DA037843 to SR and T32DA007097 (SR and others).

\section{AUTHOR CONTRIBUTIONS}

S.R. conceptualized the project. S.B., T.J., and S.R. designed experiments. S.B., G.S., F.W., J.J., U.S., L.Z., P.D., C.C., and J.D. did experiments. S.B. and S.R. wrote the manuscript.

\section{DISCLOSURE}

The authors declared no conflict of interest.

c) 2016 Society for Mucosal Immunology

\section{REFERENCES}

1. Ninković, J. \& Roy, S. Morphine decreases bacterial phagocytosis by inhibiting actin polymerization through cAMP-, Rac-1-, and p38 MAPKdependent mechanisms. Am. J. Pathol. 180, 1068-1079 (2012).

2. Roy, S. et al. Opioid drug abuse and modulation of immune function: consequences in the susceptibility to opportunistic infections. J. Neuroimmune. Pharmacol. 6, 442-465 (2011).

3. Meng, J. et al. Morphine induces bacterial translocation in mice by compromising intestinal barrier function in a TLR-dependent manner. PLoS One 8, e54040 (2013).

4. Banerjee, S. et al. Morphine induced exacerbation of sepsis is mediated by tempering endotoxin tolerance through modulation of miR-146a. Sci. Rep. 3, 1977 (2013).

5. Hilburger, M.E. et al. Morphine induces sepsis in mice. J. Infect. Dis. 176 183-188 (1997).

6. Turner, J.R. Intestinal mucosal barrier function in health and disease. Nat. Rev. Immunol. 9, 799-809 (2009).

7. Fujimura, K. \& Slusher, N. Role of the gut microbiota in defining human health. Expert Rev. Anti 8, 435-454 (2010).

8. Raybould, H.E. Gut microbiota, epithelial function and derangements in obesity. J. Physiol. 590, 441-446 (2012).

9. Power, S.E., O'Toole, P.W., Stanton, C., Ross, R.P. \& Fitzgerald, G.F. Intestinal microbiota, diet and health. Br. J. Nutr. 111, 387-402 (2014).

10. Bourzac, K. The bacterial tightrope. Nature 516, S14-S16 (2014).

11. Dawson, P.A. \& Karpen, S.J. Intestinal transport and metabolism of bile acids. J. Lipid Res. 55, 1085-1099 (2014).

12. Li, T. \& Chiang, J.Y.L. Bile acid signaling in metabolic disease and drug therapy. Pharmacol. Rev. 66, 948-983 (2014).

13. Stenman, L.K., Holma, R. \& Korpela, R. High-fat-induced intestinal permeability dysfunction associated with altered fecal bile acids. World J. Gastroenterol. 18, 923-929 (2012).

14. Stenman, L.K., Holma, R., Eggert, A. \& Korpela, R. A novel mechanism for gut barrier dysfunction by dietary fat: epithelial disruption by hydrophobic bile acids. Am. J. Physiol. Gastrointest. Liver Physiol. 304, G227-G234 (2013).

15. Sindberg, G.M. et al. An infectious murine model for studying the systemic effects of opioids on early HIV pathogenesis in the gut. J. Neuroimmune Pharmacol. 10, 74-87 (2014). 
16. Mariat, D. etal. The Firmicutes/Bacteroidetes ratio of the human microbiota changes with age. BMC Microbiol. 9, 123 (2009).

17. Jacobs, J.P. \& Braun, J. Immune and genetic gardening of the intestinal microbiome. FEBS Lett. 588, 4102-4111 (2014).

18. Breslow, J.M. et al. Potentiating effect of morphine on oral Salmonella enterica serovar Typhimurium infection is $\mu$-opioid receptor-dependent. Microb. Pathog. 49, 330-335 (2010).

19. Wang, J., Barke, R.a., Charboneau, R. \& Roy, S. Morphine impairs host innate immune response and increases susceptibility to Streptococcus pneumoniae lung infection. J. Immunol. 174, 426-434 (2005).

20. El-Hage, N., Dever, S.M., Fitting, S., Ahmed, T. \& Hauser, K.F. HIV-1 coinfection and morphine coexposure severely dysregulate hepatitis $\mathrm{C}$ virus-induced hepatic proinflammatory cytokine release and free radical production: increased pathogenesis coincides with uncoordinated host defenses. J. Virol. 85, 11601-11614 (2011).

21. Babrowski, T. et al. Pseudomonas aeruginosa virulence expression is directly activated by morphine and is capable of causing lethal gut-derived sepsis in mice during chronic morphine administration. Ann. Surg. 255, 386-393 (2012).

22. Ocasio, F.M., Jiang, Y., House, S.D. \& Chang, S.L. Chronic morphine accelerates the progression of lipopolysaccharide-induced sepsis to septic shock. J. Neuroimmunol. 149, 90-100 (2004).

23. Meng, J. et al. Opioid exacerbation of Gram-positive sepsis, induced by gut microbial modulation, is rescued by IL-17A neutralization. Sci. Rep. 5, 10918 (2015).

24. Asselin, C. \& Gendron, F.-P. Shuttling of information between the mucosal and luminal environment drives intestinal homeostasis. FEBS Lett. 588, 4148-4157 (2014).

25. Khoruts, A., Dicksved, J., Jansson, J.K. \& Sadowsky, M.J. Changes in the composition of the human fecal microbiome after bacteriotherapy for recurrent. J. Clin. Gastroenterol. 44, 354-360 (2010).

26. Khoruts, A. \& Sadowsky, M.J. Therapeutic transplantation of the distal gut microbiota. Mucosal Immunol. 4, 4-7 (2011).

27. Kelly, C.R. et al. Fecal microbiota transplant for treatment of Clostridium difficile infection in immunocompromised patients. Am. J. Gastroenterol. 109, 1065-1071 (2014).

28. Khoruts, A., Sadowsky, M.J. \& Hamilton, M.J. Development of fecal microbiota transplantation suitable for mainstream medicine. Clin. Gastroenterol. Hepatol. 13, 246-250 (2014).

29. O'Connor, W., Zenewicz, L. \& Flavell, A.R. The dual nature of $T(H) 17$ cells: shifting the focus to function. Nat. Immunol. 11, 471-476 (2010).

30. Meng, J., Li, D., Roy, S. \& Wang, F. Morphine exacerbates experimental sepsis by modulating IL17/IL22 response (IRC8P.488). J. Immunol. 192, 190.16-190.16 (2014).

31. Takahashi, N. et al. IL-17 produced by Paneth cells drives TNF-induced shock. J. Exp. Med. 205, 1755-1761 (2008).

32. Pappu, R., Rutz, S. \& Ouyang, W. Regulation of epithelial immunity by IL-17 family cytokines. Trends Immunol. 33, 343-349 (2012).

33. Ridlon, J.M., Kang, D.J., Hylemon, P.B. \& Bajaj, J.S. Bile acids and the gut microbiome. Curr. Opin. Gastroenterol. 30, 332-338 (2014).
34. Bloch, K., Berg, B. \& Rittenberg, D. The Biological Conversion of Cholesterol to cholic acid. J. Biol. Chem. 149, 511-517 (1943).

35. Jones, M.L., Tomaro-Duchesneau, C. \& Prakash, S. The gut microbiome, probiotics, bile acids axis, and human health. Trends Microbiol. 22, 306-308 (2014).

36. Jones, B.V., Begley, M., Hill, C., Gahan, C.G.M. \& Marchesi, J.R. Functional and comparative metagenomic analysis of bile salt hydrolase activity in the human gut microbiome. Proc. Natl. Acad. Sci. USA 105, 13580-13585 (2008).

37. Kumar, R., Grover, S. \& Batish, V.K. Hypocholesterolaemic effect of dietary inclusion of two putative probiotic bile salt hydrolase-producing Lactobacillus plantarum strains in Sprague-Dawley rats. Br. J. Nutr. 105, 561-573 (2011).

38. Cardona, M., Vanay, V., Midtveldt, T. \& Norin, K.E. Probiotics in gnotobiotic mice. Microb. Ecol. Health Dis. 12, 219-224 (2000).

39. Brestoff, J.R. \& Artis, D. Commensal bacteria at the interface of host metabolism and the immune system. Nat. Immunol. 14, 676-684 (2013).

40. Brenchley, J.M. \& Douek, D.C. Microbial translocation across the Gl tract. Annu. Rev. Immunol. 30, 149-173 (2012).

41. Gómez-Hurtado, I. et al. Gut microbiota dysbiosis is associated with inflammation and bacterial translocation in mice with $\mathrm{CCl} 4$-induced fibrosis. PLoS One 6, e23037 (2011).

42. Akira, S., Uematsu, S. \& Takeuchi, O. Pathogen recognition and innate immunity. Cell 124, 783-801 (2006).

43. Ding, J. \& Chang, T.L. TLR2 activation enhances HIV nuclear import and infection through Tcell activation-independent and -dependent pathways. J. Immunol. 188, 992-1001 (2012).

44. Dutta, R. et al. Morphine modulation of toll-like receptors in microglial cells potentiates neuropathogenesis in a HIV-1 model of coinfection with pneumococcal pneumoniae. J. Neurosci. 32, 9917-9930 (2012).

45. Garsin, D.A. et al. A simple model host for identifying Gram-positive virulence factors. Proc. Natl. Acad. Sci. USA 98, 10892-10897 (2001).

46. Hardy, H., Harris, J., Lyon, E., Beal, J. \& Foey, A.D. Probiotics, prebiotics and immunomodulation of gut mucosal defences: homeostasis and immunopathology. Nutrients 5 (6), 1869-1912 (2013).

47. Renga, B. et al. The bile acid sensor FXR is required for immuneregulatory activities of TLR-9 in intestinal inflammation. PLOS One 8 (1), 1-11 (2013).

48. Kakiyama, G. et al. Colonic inflammation and secondary bile acids in alcoholic cirrhosis. Am. J. Physiol. Gastrointest. Liver Physiol. 306, 929937 (2014).

49. Weingarden, A.R. et al. Microbiota transplantation restores normal fecal bile acid composition in recurrent Clostridium difficile infection. Am. J. Physiol. Gastrointest. Liver Physiol. 306, G310-G319 (2014).

50. Shaik, F.B., Prasad, D.V.R. \& Narala, V.R. Role of farnesoid $\times$ receptor in inflammation and resolution. Inflamm. Res. 64, 9-20 (2015).

51. Hamilton, M.J., Weingarden, A.R., Sadowsky, M.J. \& Khoruts, A. Standardized frozen preparation for transplantation of fecal microbiota for recurrent Clostridium difficile infection. Am. J. Gastroenterol. 107, 761-767 (2012). 